\title{
Clinical features and long-term outcome of type $A$ and type $B$ intramural hematoma of the aorta
}

\author{
Yoshimasa Moizumi, MD \\ Tsunehiro Komatsu, MDa \\ Naotaka Motoyoshi, MD \\ Koichi Tabayashi, MD ${ }^{\mathrm{b}}$
}

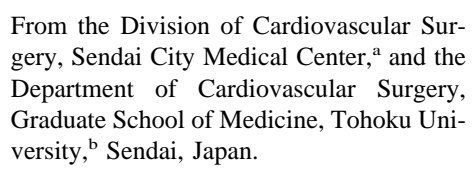

Received for publication June 19, 2003; revisions requested Sept 8, 2003; accepted for publication Sept 16, 2003.

Address for reprints: Yoshimasa Moizumi, MD, the Division of Cardiovascular Surgery, Sendai, City Medical Center, 5-22-1 Turugaya, Miyaginoku, Sendai, Miyagiken, Japan (E-mail: moizumi@openhp.or.jp).

J Thorac Cardiovasc Surg 2004;127:421-7 $0022-5223 / \$ 30.00$

Copyright () 2004 by The American Association for Thoracic Surgery

doi:10.1016/j.jtcvs.2003.09.016
Objective: Most previous reports on intramural hematoma of the aorta have focused on the initial episode. The purpose of this study was to clarify the long-term outcome of intramural hematoma of the aorta.

Methods: Ninety-four cases of intramural hematoma of the aorta (41 type A and 53 type B) were reviewed. There were 69 male and 25 female patients, and their mean age was $66.7 \pm 8.7$ years (range, $46-88$ years).

Results: Eleven (27\%) of the patients with type A hematoma and $1(2 \%)$ of the patients with type B hematoma underwent early surgical intervention. Others were treated medically, and the overall hospital mortality was $7 \%$ for patients with type versus $2 \%$ for patients with type B intramural hematomas of the aorta $(P=.315)$. Twenty-three patients, 9 (22\%) with type A and 14 (26\%) with type B intramural hematomas of the aorta, underwent late surgical intervention during the follow-up period, and there were no hospital deaths. A total of 23 patients died during the follow-up period, including 6 of intramural hematoma of the aorta-related deaths ( 3 in the type A group and 3 in the type B group). The estimated freedom from intramural hematoma of the aorta-related events at 1 and 5 years was $70 \% \pm 8 \%$ and $54 \% \pm 11 \%$ for the type A group versus $73 \% \pm 6 \%$ and $58 \% \pm 8 \%$ for the type B group, respectively $(P=.972)$. After excluding the nonintramural hematoma of the aorta-related deaths, the survival rates at 5 and 10 years were $80 \% \pm 9 \%$ and $80 \% \pm 9 \%$ for the type A group and $91 \% \pm 8 \%$ and $81 \% \pm 11 \%$ for the type B group $(P=.211)$.

Conclusions: Intramural hematoma of the aorta-related events occur equally in both types of intramural hematoma of the aorta. We recommend close follow-up for at least 5 years because most intramural hematoma of the aorta-related events occur during this period.

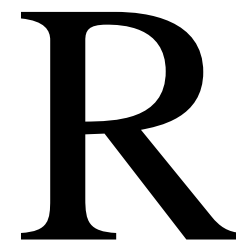

ecent advances in diagnostic modalities have enabled rapid and definitive diagnosis of intramural hematoma of the aorta (IMH), ${ }^{1-4}$ and although voluminous data on IMH have been accumulated as a result, most investigators have focused on the initial presenting episode and its management. Type B IMH is currently considered to be less severe, and medical treatment is considered appropriate. ${ }^{5}$ In 1993, Robbins and associates ${ }^{1}$ recommended that early surgical intervention be considered for all patients with type A IMH, and their recommendation was widely accepted in Western countries. ${ }^{4,5}$ Conversely, we reported that emergency surgery was reasonable in $27 \%$ of selected patients out of 33 with type A IMH, and that $50 \%$ could be managed with medical treatment alone. ${ }^{3}$

In view of the pathophysiologic process in IMH, the absence of flow and pressure communication with the aortic lumen might produce good results. ${ }^{6}$ By contrast, as Sundt ${ }^{7}$ pointed out, IMH is fundamentally a disease of the media, and thus the 
behavior of IMH is of great concern. Until now, little information has been available on the midterm to long-term outcome of IMH, ${ }^{8}$ and several questions remain. Is it true that type B IMH is less serious throughout the entire follow-up period? What is the behavior of IMH over time, and are there any differences between type A and type B IMH? Are there any predictive factors for progression of IMH? How should patients with IMH be managed on the basis of the clinical evidence?

The purpose of the present study was to clarify the clinical features and to determine the long-term outcome of type A and type B IMH by retrospectively reviewing 94 cases.

\section{Patient Population and Methods}

From January 1990 through December 2002, a total of 233 patients were referred to Sendai City Medical Center with acute aortic dissection variables. Ninety-four $(40 \%)$ of these patients were given a diagnosis of IMH. There were 69 male and 25 female patients with a mean age of $66.7 \pm 8.7$ years (range, $46-88$ years). The diagnostic criteria for IMH were previously reported ${ }^{3}$ : (1) symptoms such as severe chest or back pain like that of acute aortic dissection and (2) initial computed tomographic (CT) examination performed within 24 hours from the onset of symptoms showing the characteristic findings presented by Yamada and colleagues. ${ }^{9}$ Furthermore, the diagnosis was confirmed through operative findings or serial CT examinations.

\section{Management}

Initial management of IMH was the same, in principal, whether type A or type B IMH was found: only patients with cardiac tamponade, impending rupture, or rupture underwent surgical intervention on an emergency basis; others were treated medically, with intensive antihypertensive therapy in the intensive care unit, for a couple of days. Details of medical treatment are described elsewhere. ${ }^{3}$ After discharge, all patients were followed as outpatients, having resumed their previous lifestyles. Patients with medical treatment converted to surgical treatment in the following cases: (1) recurrence of chest or back pain suggestive of impending rupture; (2) progression to overt type A dissection; (3) progressive aortic dilatation to more than $60 \mathrm{~mm}$ in maximum diameter; or (4) progressive enlargement of the ulcer-like projection (ULP) ${ }^{10}$ to more than $20 \mathrm{~mm}$ in diameter or depth.

\section{CT Examinations}

CT images were obtained at 3- to $10-\mathrm{mm}$ intervals from the top of the aortic arch to the aortic bifurcation, with and without contrast medium. Initial CT scans were performed within 24 hours from episode onset in all patients. For medically treated patients, serial CT examinations were performed at 2 and 4 weeks after admission; at 3, 6, and 12 months after discharge; and once a year thereafter. For patients undergoing surgical intervention, follow-up CT scans were performed at discharge and then once a year. The external diameter and wall thickness of the ascending and descending aorta were measured in each slice, and the aortic arch was measured as described by Sueyoshi and coworkers. ${ }^{11}$ In type B $\mathrm{IMH}$ the maximum aortic diameter and wall thickness were rep- resented by the largest external dimension and wall thickness in the aortic arch or descending aorta.

Follow-up was obtained in all but 2 patients (98\%) by means of direct or telephone contact, communication with the primary physician, or both. The cumulative follow-up period was 415 patientyears (mean, $52.9 \pm 39.4$ months; range, 0-156 months).

Clinical features, hospital mortality, long-term survivals, and freedom from IMH-related events were compared between patients with type A and type B IMH. IMH-related events were defined as follows: (1) satisfaction of criteria for surgical conversion (with or without actual surgical intervention); (2) death from aortic rupture; (3) sudden unexplained death; and (4) progression to overt type B dissection. Furthermore, clinical characteristics and CT images were analyzed to identify predictors of IMH-related events for patients with type B IMH (Appendix).

\section{Statistical Analysis}

A software package (StatView 5.0 for Macintosh; SAS Inc, Cary, NC) was used for statistical analyses. Data are presented as means and SDs. Comparisons between type A and type B IMH were made by using the Mann-Whitney $U$ test for continuous variables. Discrete variables were treated by means of contingency tables and analyzed by using the Fisher exact test. One-, 3-, 5-, and 10-year freedoms from IMH-related events and actuarial survivals were estimated by using the Kaplan-Meier method and compared with log-rank tests. Each estimate was expressed as the mean \pm SEM. The Cox proportional hazards model was used to identify the predictors of IMH-related events throughout the entire follow-up period by using univariate and forward stepwise Cox regression analysis (with entry and removal thresholds of 0.05 and 0.1 , respectively).

\section{Results}

Among the 94 patients, 41 had type A and 53 had type B IMH. The mean ages of patients with type A and type B IMH were $67.0 \pm 9.1$ years (range, $46-88$ years) and $66.5 \pm$ 8.5 years (range, 48-84 years), respectively $(P=.789)$. Male patients were more predominant in the type B group $(85 \%)$ than in the type A group $(59 \%, P=.0051)$. Hypertension was the most common risk factor, being associated with $32(78 \%)$ patients with type A IMH and $47(87 \%)$ patients with type B IMH $(P=.256)$. IMH-related complications were more frequent in type A than in type B IMH. Twelve $(29 \%)$ patients with type A IMH but only $1(2 \%)$ patient with type B IMH were in shock on admission $(P=$ .0001). Cardiac tamponade occurred in 10 (24\%) and aortic rupture in $4(10 \%)$ patients with type A IMH, whereas aortic rupture alone occurred in only 1 patient with type B IMH. Hemiplegia occurred in 2 patients with type A IMH, but the symptoms were transient and improved after surgical intervention. Paraplegia occurred in 1 patient with type A IMH, the details of which were previously reported. ${ }^{12}$

Eleven $(27 \%)$ of 41 patients with type A IMH underwent early surgical intervention with a diagnosis of cardiac tamponade $(n=9)$ or rupture $(n=2)$. Total arch replacement was carried out in 3 patients, and ascending aorta replace- 
ment was done in 8 patients. Two of 11 patients were lost postoperatively; one died of low-output syndrome and the other of severe brain damage produced by deterioration of old cerebral infarction. Despite association of cardiac tamponade or rupture, 3 patients with type A IMH did not undergo early surgical intervention; 2 of these patients refused the operation and were treated medically, whereas another, in whom IMH was completely absorbed within a day, received medical treatment. ${ }^{13}$ Meanwhile, only 1 (2\%) patient with type B IMH underwent early surgical intervention, with a diagnosis of aortic rupture. Eighty-two (87\%) patients were initially treated medically. Five $(5 \%)$ patients required mechanical ventilation and sedation to maintain blood pressure at an appropriate level. One patient with type B IMH died of descending thoracic aortic rupture 2 days after admission. Another patient with type A IMH died of sepsis resulting from an associated liver abscess. Four of the 94 patients died (3 with type A IMH and 1 with type B $\mathrm{IMH}$ ) during the first admission, and the overall hospitality mortality was $7 \%$ in patients with type A IMH and $2 \%$ in patients with type B IMH $(P=.315)$.

Twenty-three of the 82 patients who were initially treated medically underwent surgical intervention during the follow-up period: 9 (22\%) of these patients had type A $\mathrm{IMH}$, and 14 (26\%) had type B IMH. Progression to overt type A dissection was the most common reason for surgical conversion in patients with type A IMH, and this occurred in 6 patients. In patients with type B IMH, progressive dilatation of the aorta and enlargement of the ULP were the major reasons for conversion and were seen in 5 and 9 patients, respectively. The interval between the onset of IMH and surgical conversion varied according to the cause of conversion to surgical intervention. For example, when operations were performed because of progression to overt type A dissection, the interval was $3.9 \pm 2.0$ months (range, 2-6 months). Moreover, when enlargement of ULP necessitated surgical intervention, the interval was $2.1 \pm 0.88$ months (range, 1-4 months). Conversely, when progressive dilatation of the aorta brought about surgical conversion, the interval was much longer: $23.2 \pm 19.0$ months (range, 3-49 months). Practical procedures for these lesions were varied and are listed in Table 1. There were no hospital deaths among these 23 patients. Twenty-one (51\%) patients with type A IMH and 38 (72\%) patients with type B IMH were given medical treatment alone during the entire follow-up period.

A total of 23 patients died during the follow-up period. Six patients ( 3 with type A IMH and 3 with type B IMH) died of aortic rupture or sudden unexplained death, which were considered to be IMH-related deaths. Although 2 of these 6 patients had received graft replacement previously (ascending plus arch replacement in type A IMH and descending aortic replacement in type B IMH), ruptures oc-
TABLE 1. Practical procedures and cause of surgical conversion in type $A$ and type $B$ IMH

Type A IMH Type B IMH

Progression to type A dissection

Ascending

Ascending + arch + AVR

Progression to type $B$ dissection Descending

Progression to type $A+B$ dissection

Ascending + descending

Enlargement of ulcer-like projection

Descending

Thoracoabdominal

Stent (at descending)

Impending rupture

Descending

Dilatation of thoracic aorta

Ascending + arch

Arch + descending

Thoracoabdominal

Total

$4 \quad 0$

1

0

0

1

1

0

$A V R$, Aortic valve replacement.

curred at the remaining descending thoracic aorta. In 5 of these 6 patients, marked dilatation of the descending thoracic aorta was detected before death. Two patients refused surgical intervention, and 1 patient, who was bedridden because of stroke, was judged to be beyond surgical indication. Another 2 patients were followed as outpatients because the maximum diameter of the descending aorta was less than $60 \mathrm{~mm}$. The mean interval from onset of IMH to death was 53.8 months (median, 49 months; range, 18-111 months). Seventeen other cases resulted in non-IMH-related death, and the major causes of death were cerebrovascular disease and malignancy, occurring in 3 and 2 patients with type A IMH versus 2 and 3 in patients with type B IMH, respectively. The causes of death are listed in Table 2 . The clinical course of the patients with IMH is presented as a visual flow chart in Figure 1.

The estimates of freedom from IMH-related events at 1 , 3,5 , and 10 years were $70 \% \pm 8 \%, 66 \% \pm 9 \%, 54 \% \pm$ $11 \%$, and $54 \% \pm 11 \%$ for type A IMH versus $73 \% \pm 6 \%$, $68 \% \pm 7 \%, 58 \% \pm 8 \%$, and $51 \% \pm 9 \%$ for type B IMH $(P$ $=.972$, Figure 2). Including hospital deaths, the cumulative $1-, 3-, 5-$, and 10-year survivals were $90 \% \pm 5 \%, 81 \% \pm$ $7 \%, 55 \% \pm 12 \%$, and $44 \% \pm 14 \%$ in patients with type A $\mathrm{IMH}$ versus $94 \% \pm 3 \%, 92 \% \pm 4 \%, 73 \% \pm 8 \%$, and $50 \%$ $\pm 10 \%$ in patients with type B IMH $(P=.219$, Figure 3$)$. Excluding non-IMH-related deaths, the 1-, 3-, 5-, and 10year survivals were $95 \% \pm 3 \%, 92 \% \pm 4 \%, 80 \% \pm 9 \%$, and $80 \% \pm 9 \%$ in patients with type A IMH versus $98 \% \pm$ $2 \%, 98 \% \pm 2 \%, 91 \% \pm 8 \%$, and $81 \% \pm 11 \%$ in patients with type B IMH $(P=.211$, Figure 4$)$.

Clinical profiles and CT examinations were evaluated as predictors for IMH-related events in patients with type B 
TABLE 2. Causes of the late death

\begin{tabular}{lccc}
\hline & Type A IMH & Type B IMH & Total \\
\hline Aortic rupture & 2 & 3 & 5 \\
Cerebrovascular disease & 3 & 2 & 5 \\
Malignancy & 2 & 3 & 5 \\
Myocardial infarction & 0 & 2 & 2 \\
Pneumonia & 0 & 2 & 2 \\
Renal failure & 1 & 0 & 1 \\
Anorexia nervosa & 0 & 1 & 1 \\
Sudden unexplained death & 1 & 0 & 1 \\
Accident & 0 & 1 & 1 \\
\hline Total & 9 & 14 & 23 \\
\hline
\end{tabular}

IMH. Hypertension $(P=.0046)$ and the maximum wall thickness at 2 to 4 weeks after admission $(P=.0017)$ were significantly correlated variables for IMH-related events because of enlargement of the ULP or overt type B dissection. Multivariate analysis revealed that the maximum wall thickness at 2 to 4 weeks after admission was the only predictor (hazard ratio, 1.222; 95\% confidence interval, $1.049-1.425 ; P=.01$ ). Furthermore, we calculated an optimal cutoff value of $16 \mathrm{~mm}$, resulting in positive and negative predictive values of $60 \%$ and $86 \%$, respectively.

On the other hand, the maximum external diameter on admission $(P=.001)$ and 2 to 4 weeks later $(P=.005)$ was also a significantly correlated variable for IMH-related events because of progressive aortic dilatation or rupture. Multivariate analysis revealed that the maximum external diameter on admission was a predictor (hazard ratio, 1.250; 95\% confidence interval, 1.01-1.547; $P=.04$ ), with an optimal cutoff value of $53 \mathrm{~mm}$ and positive and negative predictive values of $100 \%$ and $96 \%$, respectively (Tables 3 and 4).

\section{Discussion}

IMH develops as a result of spontaneous rupture of the vasa vasorum of the aorta ${ }^{14}$ or rupture of an atherosclerotic plaque, ${ }^{1}$ and it is considered a unique entity in aortic pathology. Although it is well known that IMH might progress or regress with time, assessment of progression or regression of IMH includes difficult problems, such as the timing of the evaluation (eg, 1 month, 1 year, and later) and the method of evaluation (clinical or radiologic). Thus on the basis of the clinical and radiologic evidence throughout the follow-up period, we substituted IMH-related events (or freedom therefrom) for progression (or regression) of IMH. In this article IMH-related events are defined as satisfaction of surgical conversion criteria or IMH-related death. It is noteworthy that the IMH-related event-free curve rapidly decreased to about $70 \%$ within 6 months and then gradually decreased to around $50 \%$ during the next 5 years, with no difference between types $\mathrm{A}$ and $\mathrm{B}$. This means that the nature of subsequent IMH-related events is variable and depends on the interval between the onset of IMH and the IMH-related event, with progression to overt dissection and progressive dilatation of the ULP, both of which are considered to be caused by intimal disruption, accounting for most of the IMH-related events within the first 6 months. In fact, all conversions to surgical treatment because of overt type A dissection or enlargement of the ULP occurred within 6 months. On the other hand, progressive aortic dilatation or aortic rupture (resulting from enlargement of the aorta without intimal disruption) accounted for most IMH-related events that occurred in both types of IMH after more than 1 year. The mean interval from onset of IMH to conversion to surgical intervention because of progressive dilatation was about 2 years, and the mean interval to death from aortic rupture was about 4 to 5 years. These results suggest that a variety of processes might be involved in the progression of IMH and that serial follow-up examinations are necessary for at least 5 years.

There have been some reports on predictive factors for the progression of type A or type B IMH. Kaji and cowork$\mathrm{ers}^{2}$ claimed that the maximum aortic diameter on the initial CT images was the only significant predictor of progression of type A IMH, with a cutoff value of $50 \mathrm{~mm}$, whereas Nishigami and associates ${ }^{15}$ reported that the maximum aortic diameter of less than $45 \mathrm{~mm}$ suggests a good prognosis of both type A and type B IMH. Sueyoshi and colleagues ${ }^{11}$ recently reported that the maximum aortic diameter and wall thickness are predictors of progression of type B IMH, with optimal cutoff values of $40 \mathrm{~mm}$ and $10 \mathrm{~mm}$, respectively. Although all of these studies found that maximum aortic diameter or wall thickness was closely related to the progression of IMH, their definitions of progression varied and included an increase in aortic diameter or wall thickness, aortic dissection, and death as a result of rupture. Because the nature of subsequent IMH-related events is variable and depends on the interval between the onset of IMH and the IMH-related event, it seemed unreasonable to lump diverse forms of progression under one name and then try to identify the predictors. In view of this, we have estimated the predictors of each IMH-related event independently. We previously reported that the only significant predictor of progression to overt type A dissection within 4 weeks was wall thickness at 2 weeks after admission, ${ }^{3}$ and in this article we defined 2 predictors in type B IMH: maximum wall thickness at 2 to 4 weeks after admission (for IMH-related events caused by enlargement of the ULP or overt type $\mathrm{B}$ dissection) and maximum aortic diameter on admission (for IMH-related events caused by progressive aortic dilatation or rupture). These findings seem obvious in a sense. A certain volume of hematoma in the aortic wall might cause the intima to become fragile and lead to intimal disruption. This might explain why wall thickness is related to progression to overt dissection or ULP. On 

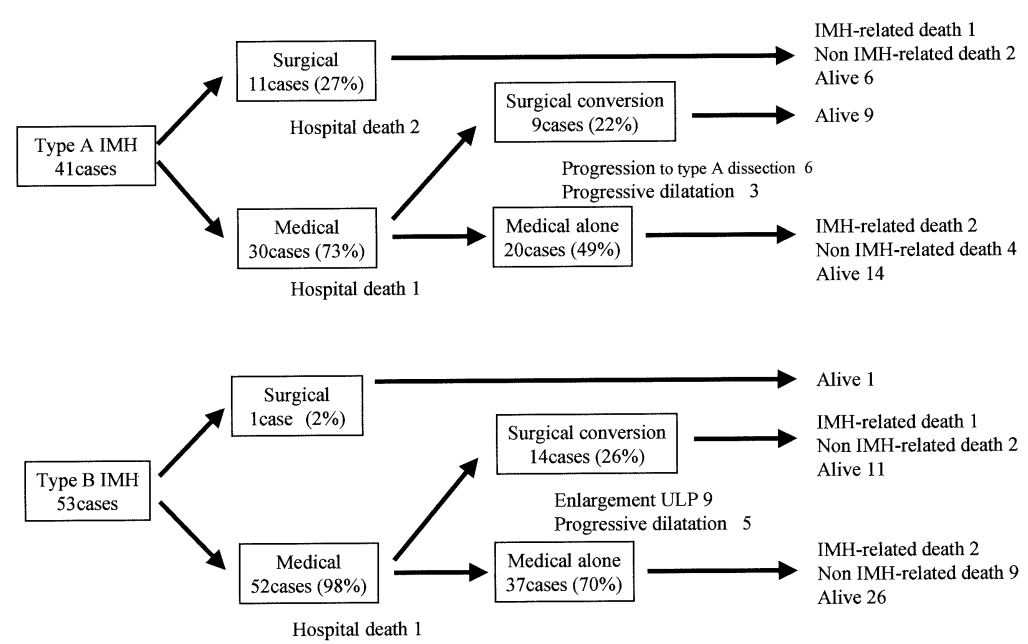

Figure 1. Flow chart of clinical course and outcomes.

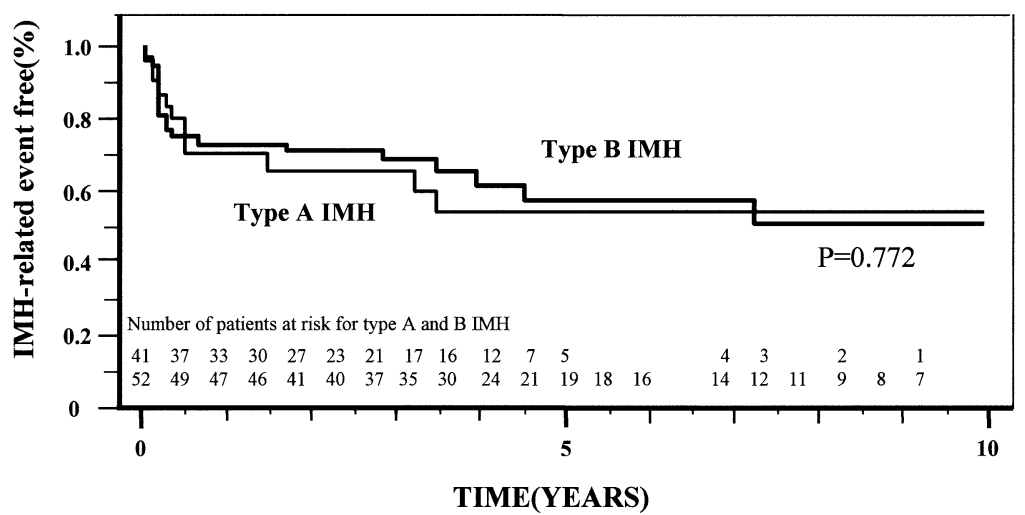

Figure 2. Freedom from IMH-related events.

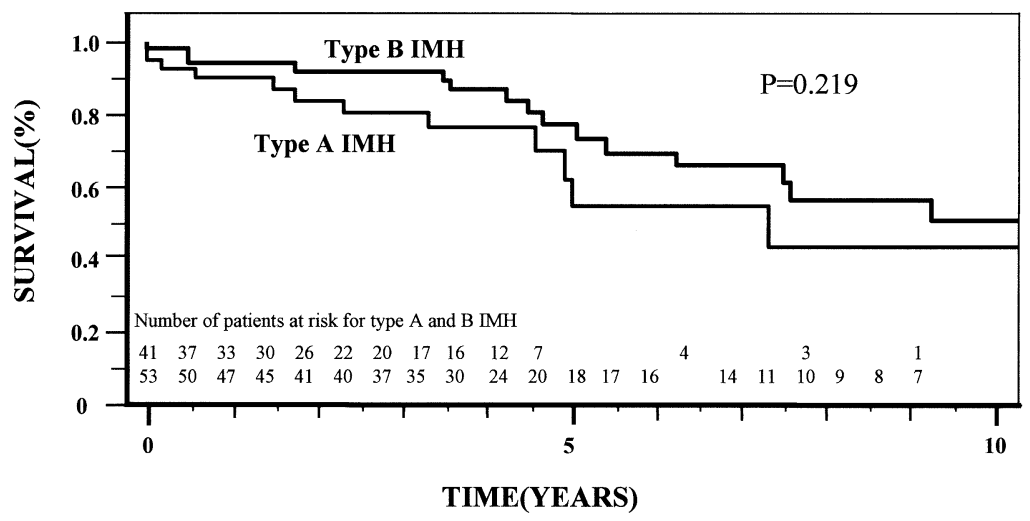

Figure 3. Cumulative survival curve.

the other hand, as Laplace's law indicates, the affected aorta is far easier to dilate in proportion to its diameter. This might also account for the relationship between aortic diameter and progressive aortic dilatation or rupture, and the growth rate has actually been reported to be 0.4 to $0.75 \mathrm{~cm} / \mathrm{y}^{8,11}$ 


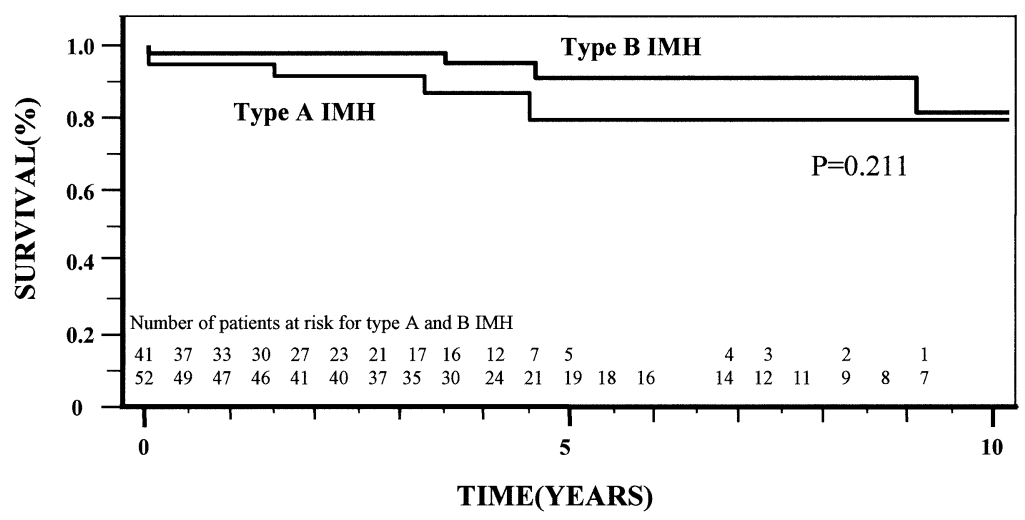

Figure 4. Survival curve excluding non-IMH-related death.

TABLE 3. The results of CT examinations

\begin{tabular}{|c|c|c|c|}
\hline IMH-related events & Yes & No & $\begin{array}{c}P \\
\text { value }\end{array}$ \\
\hline \multicolumn{4}{|l|}{$\begin{array}{l}\text { Enlargement of ULP or overt type B } \\
\text { dissection }\end{array}$} \\
\hline $\begin{array}{l}\text { Maximum aortic diameter on } \\
\text { admission (mm) }\end{array}$ & $40.7 \pm 2.6$ & $42.6 \pm 7.1$ & .406 \\
\hline $\begin{array}{l}\text { Maximum aortic diameter after } \\
2-4 \mathrm{wk}(\mathrm{mm})\end{array}$ & $43.3 \pm 5.4$ & $41.4 \pm 6.4$ & .397 \\
\hline Wall thickness on admission $(\mathrm{mm})$ & $14.4 \pm 4.7$ & $11.8 \pm 4.6$ & .115 \\
\hline Wall thickness after 2-4 wk (mm) & $14.3 \pm 4.0$ & $8.5 \pm 4.6$ & .001 \\
\hline \multicolumn{4}{|l|}{$\begin{array}{l}\text { Progressive aortic dilatation or } \\
\text { rupture }\end{array}$} \\
\hline $\begin{array}{l}\text { Maximum aortic diameter on } \\
\text { admission }(\mathrm{mm})\end{array}$ & $52.0 \pm 9.9$ & $41.1 \pm 5.0$ & .0001 \\
\hline $\begin{array}{l}\text { Maximum aortic diameter after } \\
2-4 \mathrm{wk}(\mathrm{mm})\end{array}$ & $51.0 \pm 6.8$ & $40.8 \pm 5.4$ & .0002 \\
\hline Wall thickness on admission (mm) & $13.6 \pm 7.7$ & $12.2 \pm 4.4$ & .520 \\
\hline Wall thickness after 2-4 wk (mm) & $11.2 \pm 5.3$ & $9.5 \pm 5.3$ & .490 \\
\hline
\end{tabular}

How should the patient with IMH be managed on the basis of clinical evidence? After the recommendation of Robbins and associates, ${ }^{1}$ early surgical intervention for type A IMH was widely adopted in the West and became anecdotal, without verification by clinical evidence in a large series of cases. O'Gara and DeSanctis ${ }^{5}$ asserted that IMH should be managed according to the same guidelines as classic dissection; that is, if unassociated with serious complications, type B IMH should be managed medically, like classic type B dissection, but type A IMH requires urgent surgical repair. On the basis of the midterm follow-up of IMH, Tittle and associates ${ }^{8}$ have recommended surgical repair regardless of the lesion involved because of the high early rupture rate and the documented occurrence of late rupture. Our results do not necessarily support their recommendations. As stated above, we found that early surgical intervention is indispensable in around $30 \%$ of patients with type A IMH and $2 \%$ of patients with type B IMH but that about $50 \%$ of patients with type A IMH and $70 \%$ of patients with type B IMH could be managed medically alone. Moreover, all late ruptures occurred in the descending aorta, and 5 of the 9 patients whose treatment was converted to surgical intervention required arch or descending aortic procedures, even for type A IMH. Accordingly, patient selection is primary and prophylactic surgical intervention, which can reduce the risk of death and shorten the hospital stay and can be used to extract anticipated patients. Indeed, after identifying a predictor of progression to type A dissection, we decided to perform prophylactic ascending aortic replacement in patients with type A IMH with a wall thickness of greater than $12 \mathrm{~mm}$. As predictors of other IMHrelated events (progressive aortic dilatation or fatal rupture) have not yet been determined to be predictors of type A IMH, serial CT examinations are necessary to avoid missing the opportunity for surgical treatment. IMH-related events also occurred during the follow-up period in about $50 \%$ of patients with type B IMH. Although predictors of IMHrelated events in patients with type B IMH have been identified, they are not always available in practice. Because it is always difficult to accurately forecast the location of the ULP or the extent of dilatation of the aorta at the time of the first admission, prophylactic procedures should not be done at random. Under these circumstances, closed and serial imaging examinations are of overriding importance.

By taking appropriate measures, we managed patients with type A or type B IMH by using common criteria. The result was no significant difference between patients with type A and type B IMH in hospital mortality; in 1-, 3-, 5-, or 10-year cumulative survival; or in actuarial freedom from IMH-related events. The cumulative survival showed a downward trend after 5 years and decreased to less than $50 \%$ at 10 years, but this seemed to reflect the advanced age of the patients with IMH. After excluding non-IMH-related deaths, the 10-year survivals in both types of IMH remained greater than $80 \%$. 
TABLE 4. Results of univariate and multivariate analysis for progression of IMH in type B IMH

\begin{tabular}{|c|c|c|c|c|c|}
\hline \multirow[b]{2}{*}{ Variable } & \multicolumn{2}{|c|}{ Univariate } & \multicolumn{3}{|c|}{ Multivariate } \\
\hline & $\chi^{2}$ & $P$ value & Hazard ratio & 95\% confidence interval & $P$ value \\
\hline \multicolumn{6}{|c|}{ Enlargement of ULP or overt dissection } \\
\hline $\begin{array}{l}\text { Hypertension } \\
\text { CT examination 2-4 wk }\end{array}$ & 8.050 & .0046 & 3.003 & $0.681-13.24$ & .146 \\
\hline Maximum diameter & 0.651 & .420 & & & \\
\hline Wall thickness & 9.852 & .0017 & 1.222 & $1.049-1.425$ & .010 \\
\hline \multicolumn{6}{|c|}{$\begin{array}{l}\text { Aortic dilatation or rupture } \\
\text { CT examination on admission }\end{array}$} \\
\hline $\begin{array}{l}\text { Maximum diameter } \\
\text { Wall thickness }\end{array}$ & $\begin{array}{r}10.308 \\
0.849\end{array}$ & $\begin{array}{l}.0013 \\
.357\end{array}$ & 1.250 & $1.01-1.547$ & .040 \\
\hline \multicolumn{6}{|c|}{ CT examination 2-4 wk later } \\
\hline Maximum diameter & 8.058 & .005 & 1.103 & $0.917-1.327$ & .297 \\
\hline Wall thickness & 0.012 & .913 & & & \\
\hline
\end{tabular}

Finally, 2 special points should be emphasized. First, 2 patients died of aortic rupture despite having a maximum aortic diameter of less than $60 \mathrm{~mm}$ just before death. This indicates that the appropriate size criterion for surgical repair should be reconsidered. Second, 2 other patients died of aortic rupture in spite of a previous graft replacement, indicating that surgical intervention cannot completely prevent late rupture and that serial CT examinations are necessary, even after an operation.

\section{Conclusions}

IMH-related events occur over time in about $50 \%$ of patients with IMH, and most of them occur within 5 years. Although predictors of IMH-related events in type B IMH have been identified, it is difficult to forecast the location of the ULP or the extent of dilatation of the aorta, and closed and serial CT examinations are of overriding importance. After excluding non-IMH-related deaths, the 5- and 10-year survivals in patients with both types of IMH remained greater than $80 \%$. By taking appropriate measures, IMH, regardless of location, can be managed on the basis of uniform criteria.

We thank Mr Takenobu Tasaki for valuable help with statistical analysis.

\section{References}

1. Robbins RC, McManus RP, Mitchell RS, Latter DR, Moon MR, Olinger GN, et al. Management of patients with intramural hematoma of the thoracic aorta. Circulation. 1993;88(suppl II):II1-10.

2. Kaji S, Nishigami K, Akasaka T, Hozumi T, Takagi T, Kawamoto T, et al. Prediction of progression or regression of type A aortic intramural hematoma by computed tomography. Circulation. 1999 100(suppl II):II281-6.

3. Moizumi Y, Komatsu T, Motoyoshi N, Tabayashi K. Management of patients with intramural hematoma involving the ascending aorta. J Thorac Cardiovasc Surg. 2002;124:918-24.

4. Maraj R, Rerkpattanapipat P, Jacobs LE, Makornwattana P, Kotler MN. Meta-analysis of 143 reported cases of aortic intramural hematoma. Am J Cardiol. 2000;86:664-8.
5. O'Gara PT, DeSanctis RW. Acute aortic dissection and its variants: toward a common diagnostic and therapeutic approach. Circulation. 1995;92:1376-8.

6. Kang DH, Song JK, Song MG, Lee IS, Song H, Lee JW, et al. Clinical and echocardiographic outcomes of aortic intramural hemorrhage compared with acute aortic dissection. Am J Cardiol. 1998;8:202-6.

7. Sundt TM. Management of intramural hematoma of the ascending aorta: still room for debate. J Thorac Cardiovasc Surg. 2002;124: 894-5.

8. Tittle SL, Lynch RJ, Cole PE, Singh HS, Rizzo JA, Kopf GS, et al. Midterm follow-up of penetrating ulcer and intramural hematoma of the aorta. J Thorac Cardiovasc Surg. 2002;123:1051-9.

9. Yamada T, Tada S, Harada J. Aortic dissection without intimal rupture: diagnosis with MR imaging and CT. Radiology. 1988;168:347-52.

10. Tisnado J, Cho SR, Beachley MC, Vines FS. Ulcerlike projections: a precursor angiographic sign to thoracic aortic dissection. AJR Am J Roentgenol. 1980;135:719-22.

11. Sueyoshi E, Imada T, Sakamoto I, Matsuoka Y, Hayashi K. Analysis of predictive factors for progression of type B aortic intramural heatoma with computed tomography. J Vasc Surg. 2002;35:1179-83.

12. Motoyoshi N, Komatsu T, Moizumi Y, Tabayashi K. Atypical paraplegia following aortic intramural hematoma. $J$ Thorac Cardiovasc Surg. 2003;125:409-10.

13. Ohmi M, Tabayashi K, Moizumi Y, Komatsu T, Sekino Y, Goko C. Extremely rapid regression of aortic intramural hematoma. $J$ Thorac Cardiovasc Surg. 1999;118:968-9.

14. Gore I. Pathogenesis of dissecting aneurysm of the aorta. Arch Pathol Lab Med. 1952;53:142-53.

15. Nishigami K, Tsuchiya T, Shono H, Horibata Y, Honda T. Disappearance of aortic intramural hematoma and its significance to the prognosis. Circulation. 2000;102(suppl III):III243-7.

\section{Appendix}

\section{Clinical characteristics and CT image variables analyzed.}

Sex

Age

Diabetes mellitus

Hypertension

Hyperlipidemia

Maximum external diameter on admission

Maximum external diameter at 2 to 4 weeks after admission

Maximum wall thickness on admission

Maximum wall thickness at 2 to 4 weeks after admission 